\title{
Determining the Shear Strength Properties of a Soil-geogrid Interface Using a Large-scale Direct Shear Test Apparatus
}

\author{
Jakub Stacho ${ }^{1 *}$, Monika Sulovska1', Ivan Slavik ${ }^{1}$ \\ ${ }^{1}$ Department of Geotechnics, Faculty of Civil Engineering, University of Technology in Bratislava, Radlinskeho 11, 81005 Bratislava, \\ Slovakia \\ * Corresponding author, e-mail: jakub.stacho@stuba.sk
}

Received: 14 February 2020, Accepted: 04 June 2020, Published online: 27 July 2020

\begin{abstract}
The paper deals with the laboratory testing of coarse-grained soils that are reinforced using a geogrid. The shear strength properties were determined using a large-scale direct shear test apparatus. The tests were executed on original as well as on reinforced soil, when the geogrid was placed on a sliding surface, which permitted determining the shear strength properties of the soil-geogrid interface. The aim of the tests was to determine the interface shear strength coefficient $\alpha$, which represents the ratio of the shear strength of the soil-geogrid interface to the unreinforced soil. The tests were executed on 3 samples of coarse-grained materials, i.e., poorly graded sand, poorly graded fine gravel and poorly graded medium gravel. Two types of geogrids were tested, i.e., a woven polyester geogrid and a stiff polypropylene geogrid. The results of the laboratory tests on the medium gravel showed that the reduction coefficient $\alpha$ reached higher values in the case of the stiff polypropylene geogrid. In the cases of the fine gravel and sand, the values of the interface coefficient $\alpha$ were similar to each other. The shear strength of the interface was reduced or was similar to the shear strength of unreinforced soil in a peak shear stress state, but significantly increased with horizontal deformations, especially for the fine gravel and sand. The largest value of the coefficient $\alpha$ was measured in the critical shear stress state. Based on the results of the testing, a correlation which allows for determining the optimal grain size distribution was obtained.
\end{abstract}

Keywords

soil improvement, soil reinforcement, geosynthetics, geogrid, interface shear strength

\section{Introduction}

Soil reinforcement using a geosynthetic belongs among the most often-used methods of soil improvement or stabilization. Geosynthetics can be applied to mechanically stabilized embankments and earth walls, which are composite structures made of geogrid layers and compacted soil or backfill. Examples of these structures are road and railway embankments, e.g., [1-7], and reinforced retaining walls, e.g., [8, 9]. Geosynthetics can be suitably used to improve resistance and reduce the settlement of shallow foundations, e.g., [10].

The stability and effectiveness of these constructions mostly depend on the interaction between the original soil and the geogrid. Based on the method used of the geogrid, the failure mechanism of reinforced earth structures consists of pull-out failure and sliding along the reinforcement [11]. The bearing capacity of the reinforcement for pulling out of the soil can be determined using a pull-out test. The test can be done using a physical model on an adequately reduced scale or using an actual scale field test, see e.g., [12]. A failure caused by the soil sliding on the interface with a geogrid depends on the shear strength of the soil-geogrid interface, which can be determined using a large-scale direct shear test apparatus, e.g., [11, 13-18]. The reduction or increase in the shear strength properties of the soil-geogrid interface is given by the coefficient $\alpha$, which represents the ratio of the shear strength of the soil-geogrid interface and the shear strength of the original unreinforced soil, e.g., [19].

Much published research has already focused on determining the shear strength coefficient $\alpha$ of the interface. The values of the coefficient $\alpha$ depended on the material tested and the type of geogrid. The results of the conventional direct shear tests published by [13] showed that the use of a polyester yarn geogrid with different types of soils lead to determining the coefficient $\alpha$ in the range of $0.89-1.01$. The use of a polypropylene geogrid in fine sandy 
and gravelly soils allows for determining the coefficient $\alpha$ in a range of 0.94-1.12 [20,21], but in the case of a crusher run, the coefficient $\alpha$ can decrease to 0.767-0.94 [22]. Sweta and Hussaini [23] stated that the coefficient $\alpha$ also depends on the size of the aperture of the geogrid and the grain size of the soil tested, when the optimum ratio of the geogrid's open area to the grain size $d_{50}$ is within a range of $0.95-1.54$. The values of the coefficient $\alpha$ published were within a range of $0.86-1.06$. The use of different types of woven black geogrids always causes a reduction in the coefficient $\alpha$ [24]. Different types of materials such as recycled concrete aggregate, crushed brick, and reclaimed asphalt pavement, reinforced using a polypropylene geogrid, were tested by [25]. They prepared "non-conventional" shear strength tests, where the geogrid was placed about $7 \mathrm{~mm}$ above the sliding surface, which caused an increase of about 1.8 times in the coefficient $\alpha$ in comparison to conventional shear strength tests. The materials they tested were similar to quarry stone and had a high initial shear strength, i.e., $\tau_{0}{ }^{\prime}=30-114 \mathrm{kPa}$, which was also reflected in the results and differences in the interface's shear strengths between the conventional and non-conventional tests.

An increase or decrease of the soil-geogrid interface's shear strength in comparison to the shear strength of the original unreinforced soil depends on the following parameters: the internal shear strength of the soil within the geogrid's open area; the shear strength (friction) between the soil and the surface of the geogrid; the passive resistance between the soil and the transverse ribs, e.g. [26]. It can be assumed that the final value of the coefficient $\alpha$ is a function of the grain size distribution of the material tested and the size (geometry) of the geogrid tested. The density of the soil tested also has a significant impact on the soil-geogrid interface's shear strength, but it is lacking in the results of much research, which makes it difficult to compare the results of different authors.

The properties of the soil-geogrid interface's shear strength are included in the analytical design, e.g. [26], as well as the numerical modeling of reinforced earth structures. The numerical modeling allows for the modeling of geogrids with interface elements on their surfaces, which define the shear strength of the soil-geogrid interface, e.g., [27, 28].

The paper presents the results and analysis of the soil-geogrid interface's shear strength for three types of coarse-grained soils (poorly graded sand, poorly graded fine gravel and poorly graded medium gravel), reinforced with two different types of geogrids (a stiff polypropylene geogrid and a soft woven polyester geogrid). The tests were executed according to a conventional method, using a large-scale direct shear test apparatus. The aim of the study is to determine the coefficient $\alpha$ and provide an analysis, which makes recommendations for the optimal and effective design of the types of geogrids tested.

\section{Properties of the soils tested}

Well graded gravel with a low sand content is the most optimal soil for soil reinforcement using the selected types of geogrids. The soil usually consists of fractions of $0.125-31.5 \mathrm{~mm}$. This type of soil is easy to compact and has high values of shear strength and deformation properties, e.g., [29]. However, the soil seems to be unsuitable for the presented study, because after its reinforcement by the geogrid and its compaction, it is very difficult to obtain its state, when the same content of sandy and gravel fractions is in the contact with the geogrid. Depending on the proportion of sandy and gravel fractions, different shear strengths will be determined, which further complicates an analysis of the results.

For this reason, three samples of coarse-grained soils with different grain sizes were prepared; each sample consisted of only two fractions. This allowed for the easy homogenization of the sample and resulting state, when grains of approximately the same size were contact with the reinforcement during the shear tests. The grain size distribution curves and photos of the tested samples are shown in Fig. 1.

Sample 1 consists of an 8-32 mm fraction. Based on the standard STN 72 1001:2010 (Classification of soil and rock), the sample was classified as poorly graded medium gravel (GPm). The diameters of the grains for the selected percentile as well as the uniformity coefficient and

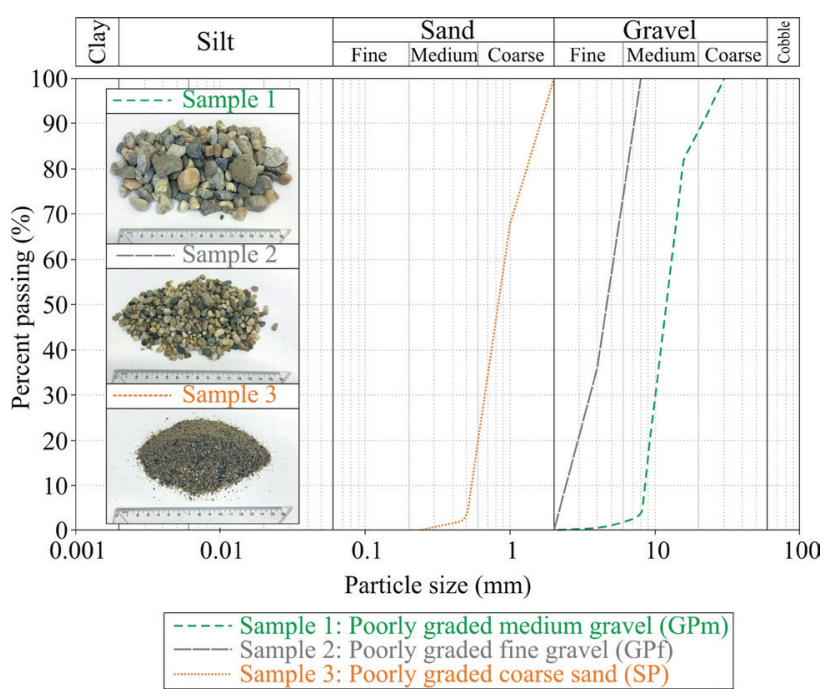

Fig. 1 Grain-size distribution curves of the soils tested 
Table 1 Properties of the soils tested

\begin{tabular}{lccccc}
\hline Parameter / Property & & Unit & Sample 1 & Sample 2 & Sample 3 \\
\hline & $d_{10}$ & $\mathrm{~mm}$ & 8.7 & 2.6 & 0.56 \\
Diameter of grain for & $d_{30}$ & $\mathrm{~mm}$ & 10.7 & 3.7 & 0.71 \\
$\begin{array}{l}\text { selected percentile } \\
d_{50}\end{array}$ & $\mathrm{~mm}$ & 12.7 & 4.9 & 0.86 \\
& $d_{60}$ & $\mathrm{~mm}$ & 13.7 & 5.5 & 0.94 \\
$\begin{array}{l}\text { Uniformity } \\
\text { coefficient }\end{array}$ & $c_{u}$ & - & 1.58 & 2.16 & 1.68 \\
$\begin{array}{l}\text { Coefficient of } \\
\text { gradation }\end{array}$ & $c_{c}$ & - & 0.96 & 0.97 & 0.96 \\
Minimal bulk density & $\rho_{\min }$ & $\mathrm{kg} . \mathrm{m}^{-3}$ & 1527.4 & 1620 & 1448.1 \\
Maximal bulk density & $\rho_{\max }$ & $\mathrm{kg} \cdot \mathrm{m}^{-3}$ & 1799.1 & 1823.3 & 1751.1 \\
\hline
\end{tabular}

coefficient of gradation are presented in Table 1. Sample 2 consists of a $2-8 \mathrm{~mm}$ fraction, classified as poorly graded fine gravel (GPf). Sample 3 consists of a $0.5-2 \mathrm{~mm}$ fraction, classified as poorly graded coarse sand (SP). The index properties of the soils tested are given in Table 1. Determining the minimal and maximal bulk density was done using conventional laboratory tests, as presented by, e.g., [30], which allowed for determining the density index $\left(I_{D}\right)$ for each shear test executed.

\section{Properties of the geogrids tested}

The tests were executed with two different types of geogrids, i.e., TenCate Miragrid GX55/30 and Thrace TG3030S. The GX55/30 reinforcement is a woven geogrid made of polyester (PET) with polymeric UV surface protection. The geogrid can be used for reinforcing retaining walls, steep slopes, and embankments as well as improving the shear and deformation parameters of the subsoil under an embankment or a shallow foundation. The geogrid is composed of high modulus polyester fibers suitable for the application of short and long-term soil reinforcement [31]. The main properties of the geogrid are stated in Table 2. The TG3030S reinforcement is a biaxial stiff polypropylene (PP) geogrid, which is made using the extrusion method of punching a pattern of holes, followed by stretching in both directions under a controlled temperature. The geogrid is suitable for the reinforcement of retaining walls and steep slopes as well as the reinforcement of the subsoil under embankments or contaminated soil [32]. The main properties of the geogrid are stated in Table 2.

The geogrid is not stressed to a great extent during the direct shear test within the normal stresses used in the study presented. For this reason, the tensile strengths only have a small impact on the shear strength of the soil-geogrid interface, which was already presented by, e.g., [13]. The most important properties of the geogrids in relation to the shear strength of the interface are the open size area
Table 2 Properties of the soils tested

\begin{tabular}{|c|c|c|c|}
\hline \multirow{2}{*}{$\begin{array}{l}\text { Type of geogrid } \\
\text { Parameter / Property }\end{array}$} & \multirow[b]{2}{*}{ Unit } & \multirow{2}{*}{$\begin{array}{l}\text { GX55/30 } \\
\text { Woven } \\
\text { "Soft" }\end{array}$} & \multirow{2}{*}{$\begin{array}{c}\text { TG3030S } \\
\text { Polypropylene } \\
\text { "Stiff" }\end{array}$} \\
\hline & & & \\
\hline $\begin{array}{l}\text { Tensile strength - machine } \\
\text { direction }\end{array}$ & $\mathrm{kN} \cdot \mathrm{m}^{-1}$ & 58 & 30 \\
\hline Tensile strength - cross direction & $\mathrm{kN} \cdot \mathrm{m}^{-1}$ & 55 & 30 \\
\hline $\begin{array}{l}\text { Minimal tensile strength - } \\
\text { machine direction }\end{array}$ & $\mathrm{kN} \cdot \mathrm{m}^{-1}$ & 30 & 11 \\
\hline $\begin{array}{l}\text { Minimal tensile strength - cross } \\
\text { direction }\end{array}$ & $\mathrm{kN} \cdot \mathrm{m}^{-1}$ & 25 & 11 \\
\hline $\operatorname{Mesh}$ size $\left(L_{\text {geogrid }}\right)$ & $\mathrm{mm}$ & $25 \times 25$ & $40 \times 40$ \\
\hline Thickness $\left(h_{\text {geogrid }}\right)$ & $\mathrm{mm}$ & 1.0 & 2.0 \\
\hline Area of geogrid (closed-size area) & $\%$ & 29.44 & 22.57 \\
\hline Open-size area & $\%$ & 70.56 & 77.43 \\
\hline
\end{tabular}

and the thickness of the geogrid (especially the thickness of the transverse ribs). These properties for the geogrids tested are presented in Table 2.

\section{Preparation of the shear test}

The direct shear tests were executed using a large SHEARMATIC 27-WF 2304 direct shear test apparatus. The ground plan dimensions of the shear box are $300 \times 300 \mathrm{~mm}$, and the height is equal to $200 \mathrm{~mm}$. The apparatus is fully automated, and the following parameters were recorded during the test: normal (vertical) stress, shear force, horizontal and vertical deformation. Each test consists of two phases: the consolidation phase and the shear test phase. The tests were done for normal stresses, i.e., $\sigma_{n}=50,100$ and $150 \mathrm{kPa}$. The actual values of the normal stresses recorded using the sensors were a little lower. The times selected for the consolidation phase were about 30 mins for Samples 1 and 2 and about 120 mins for Sample 3. Steady deformations were ensured before the shear tests. The horizontal movement was equal to $1 \mathrm{~mm} \cdot \mathrm{min}^{-1}$ for Samples 1 and 2, and a $0.5 \mathrm{~mm} \cdot \mathrm{min}^{-1}$ for Sample 3. The maximal horizontal movement in all the cases was about $60 \mathrm{~mm}$. The scheme of the apparatus is shown in Fig. 2. The movable part is an external box (container) with a lower shear box. The upper part of the box is linked to a load cell for recording the shear force.

The tests were firstly executed for the original unreinforced soil samples. Subsequently, the geogrid was fixed to the upper part of the box, and the tests were repeated. The fixation of the geogrid (geosynthetics) to the upper part of the shear box is shown in Fig. 2. The same volume of soil was used for both the unreinforced and reinforced samples. The tests for the reinforced soil were according to the following procedure: 
- the soil was compacted in the lower part of the box up to the upper edge, where the sliding surface is (compacted in 2 layers of about $5 \mathrm{~kg}$ )

- the upper part of the box with a fixed geogrid was placed on the lower part of the box

- another $5 \mathrm{~kg}$ of soil was added to the box; using slight vibrations, the state when the grains were sufficiently wedged in the geogrid was achieved. The soil layer, which represents the middle part of the soil layer with the geogrid was subsequently compacted

- two layers of soil of approximately $5 \mathrm{~kg}$ were compacted in the upper part of the box.

The placement of the upper part of the box with the geogrid on the lower part of the box before adding the soil is shown in Fig. 3.

\section{Results of the testing}

The first series of tests were done using Sample 1 (GPm). The weight of the soil used was about $25.1 \mathrm{~kg}$. The minimal bulk density was equal to $1527 \mathrm{~kg} . \mathrm{m}^{-3}$, and the maximal density was equal to $1799 \mathrm{~kg} \cdot \mathrm{m}^{-3}$. These values allowed for determining the $I_{D}$ of the sample after its compaction in the shear box. The value of $I_{D}$ was within a range of $0.70-0.73$.

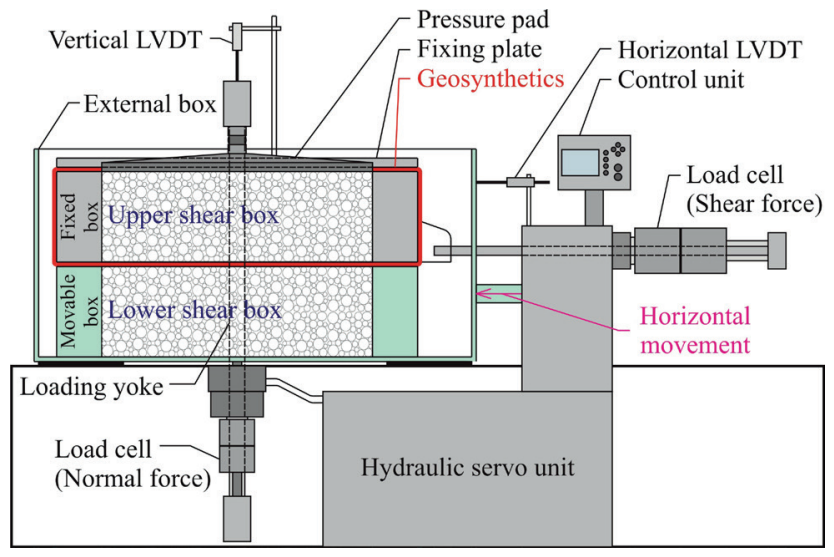

Fig. 2 Cross-section scheme of the large-scale direct shear test apparatus
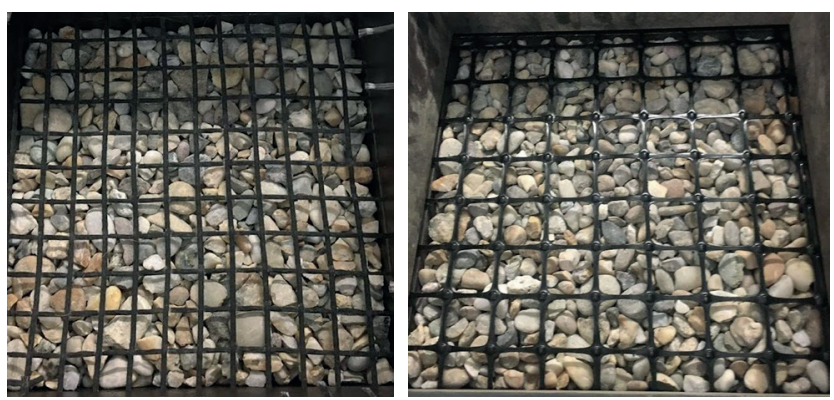

Fig. 3 Fixation of the geogrid in the shearing area before placing and compacting Sample 1 into the upper part of the box - GX55/30 (left) and TG3030S (right)
The results are presented for 3 loading stages, i.e., normal stresses of $\sigma_{1}=50 \mathrm{kPa}, \sigma_{2}=100 \mathrm{kPa}, \sigma_{3}=150 \mathrm{kPa}$. The shear stress curves for the unreinforced soil sample are shown in Fig. 4, left. The dependence of the vertical deformation on the horizontal movement is shown in Fig. 4, right. It can be seen that the behavior of the sample is dilating. The maximum value of the vertical deformation is about $8-9 \mathrm{~mm}$, depending on the horizontal movement.

In the next part of the test, the GX55/30 geogrid was installed, and the tests were repeated. The shear strength of the soil-geogrid interface was about $10 \%$ smaller than the unreinforced shear strength, except the critical shear strength for the loading stage $\sigma_{2}$, when the reinforced and unreinforced shear strengths were close to each other. The soil-geogrid interface's shear strengths were more similar to the unreinforced shear strength in the case of the TG3030S geogrid. The peak values of the interface's shear strength were a little bit lower than the unreinforced ones in the cases of normal stresses $\sigma_{1}$ and $\sigma_{3}$, but in the case of the normal stress $\sigma_{2}$, the value was a little bit higher. The critical values of the shear strengths were very similar to each other. The use of both geogrids in Sample 1 did not result in any significant changes in the vertical deformations in comparison to the unreinforced sample (Fig. 4, right).

The second series of tests were done using Sample 2 (GPf). The weight of the soil used was about $25.0 \mathrm{~kg}$. The minimal bulk density was equal to $1620 \mathrm{~kg} . \mathrm{m}^{-3}$, and the maximal one was equal to $1823 \mathrm{~kg} \cdot \mathrm{m}^{-3}$. The value of $I_{D}$ was within a range of $0.93-0.98$ during the testing. The shear strength curves of the unreinforced and reinforced samples are presented in Fig. 5. The soil-geogrid interface's shear strength curves were very similar for both geogrids used. In the peak stress state, the interface's shear strengths were a little lower than the unreinforced ones. On the contrary, in the critical stress state, the interface's shear strengths were higher than the shear strengths of the unreinforced sample. The vertical deformations were also very similar for the unreinforced and reinforced soil samples. The dilation was in a range of about 3-4 $\mathrm{mm}$.

The last series of tests were done using Sample 3 (SP). The weight of the tested sample was about $22.0 \mathrm{~kg}$. The minimal bulk density was equal to $1448 \mathrm{~kg} \cdot \mathrm{m}^{-3}$, and the maximal one was equal to $1751 \mathrm{~kg} \cdot \mathrm{m}^{-3}$. The value of $I_{D}$ was within a range of $0.98-0.99$. The shear strength curves of the unreinforced and reinforced samples are presented in Fig. 6, left. The results of the measurements show that the peak shear strengths of the unreinforced 

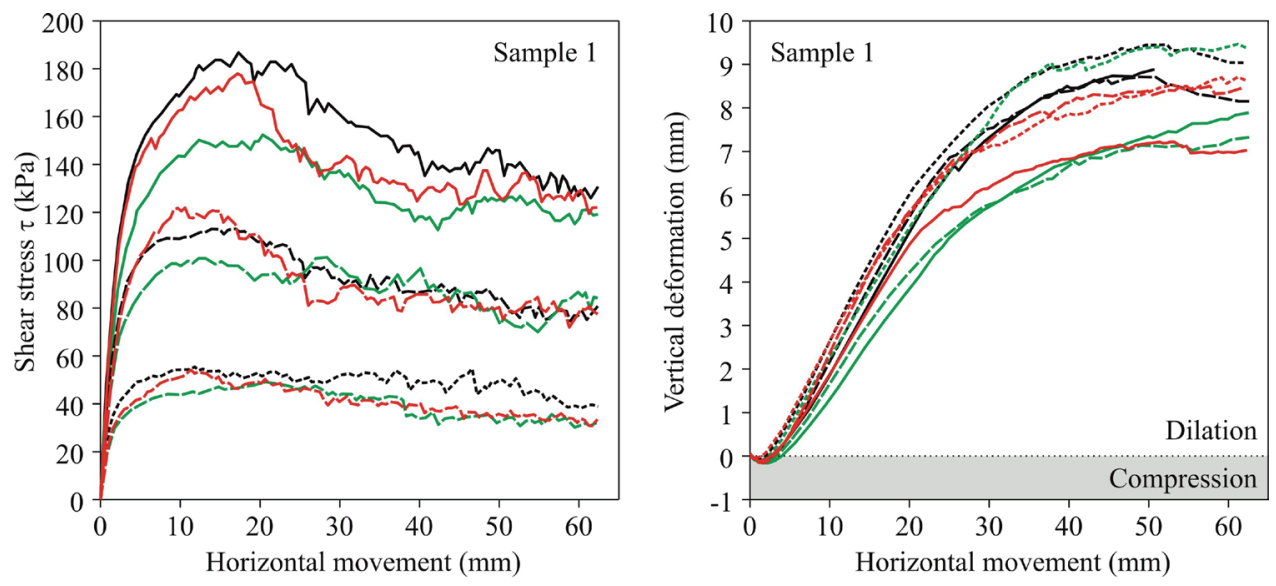

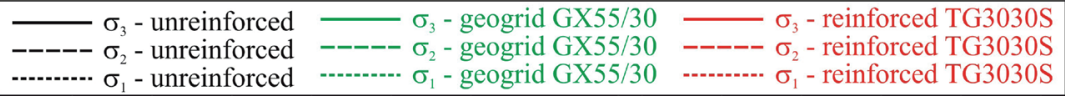

Fig. 4 Results of the shear test of Sample 1
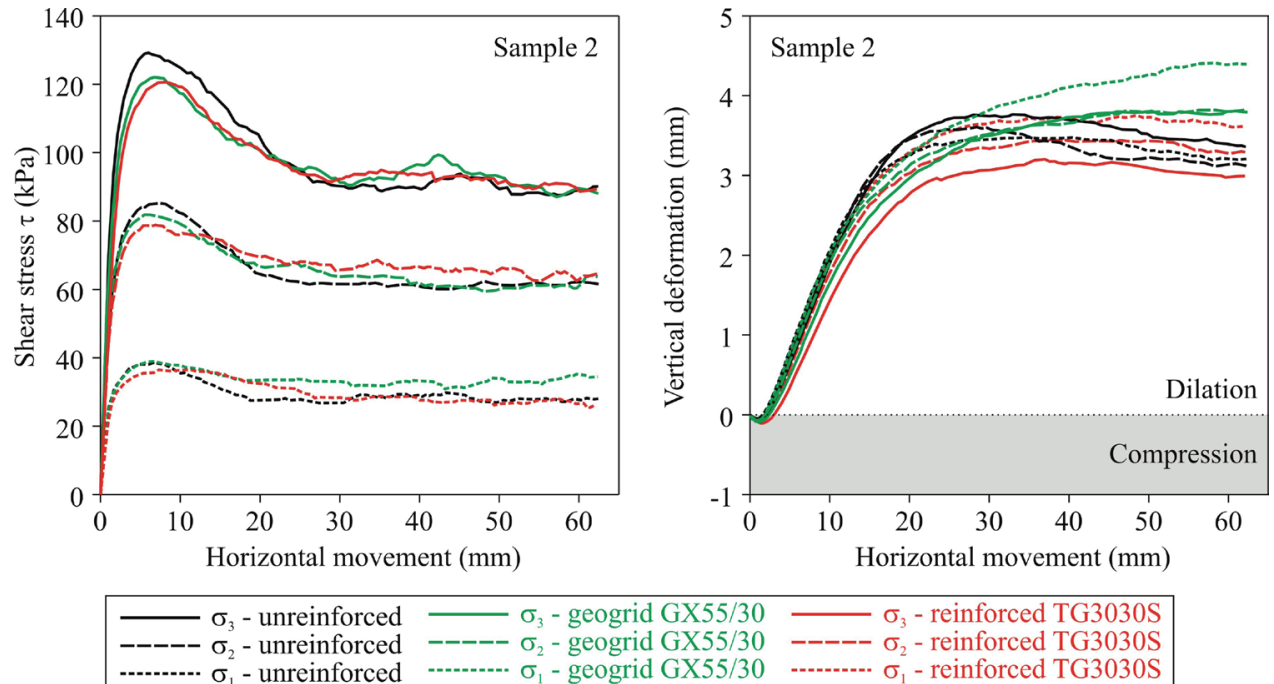

Fig. 5 Results of the shear test of Sample 2
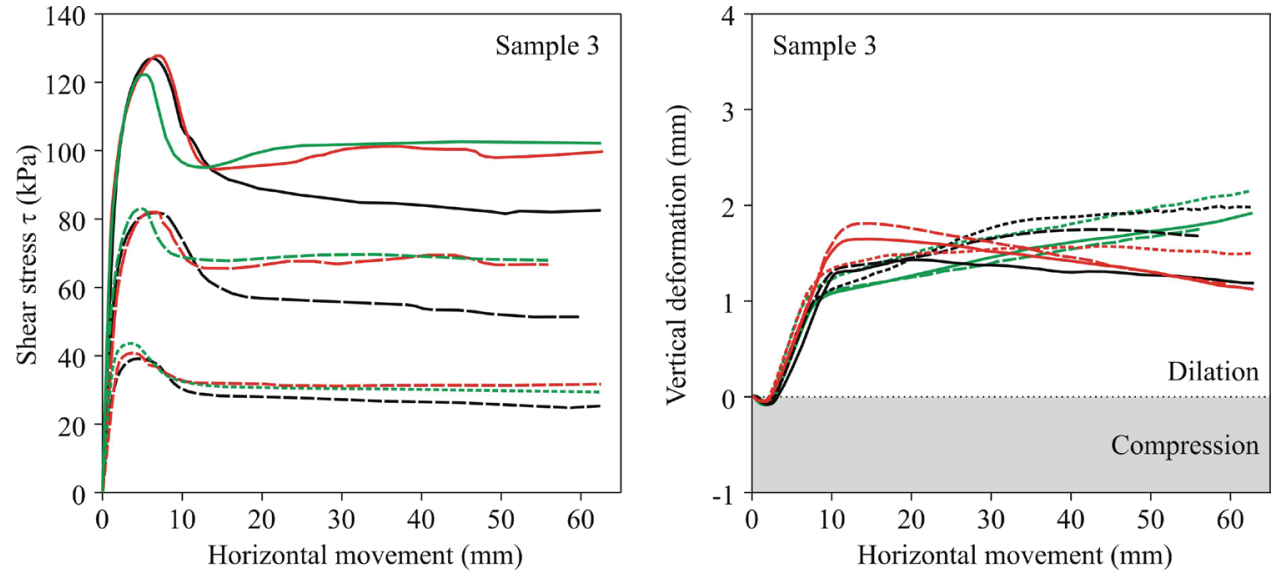

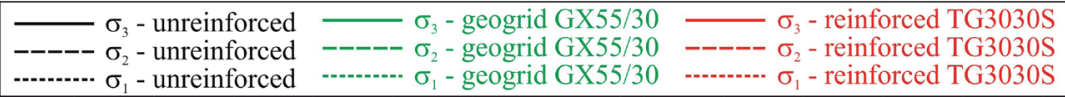

Fig. 6 Results of shear test of Sample 3 
and reinforced samples are very similar to each other. Significant differences were obtained for the critical shear strength. The interface's shear strength was higher than the shear strength of the original soil. The dilation measured was within a range of 1-2 mm (Fig. 6, right).

The parameters of the shear strength are presented in Table 3, where $\varphi_{p}{ }^{\prime}$ and $\varphi_{c}{ }^{\prime}$ are the peak and critical angles of the shear strength; $\tau_{0, p}{ }^{\prime}$ and $\tau_{0, c}{ }^{\prime}$ are the peak and critical initial shear strengths. It can be stated that the peak angle of shear strength is composed of the critical angle of shear strength and the portion due to dilation behavior, see e.g. [33]. The ratio between the interface's shear strength and the unreinforced shear strength is quantitatively represented by the interface's shear strength coefficient a according to the following equation:

$$
\alpha=\tau_{\text {soil-geogrid }} / \tau_{\text {soil }},
$$

where $t_{\text {soil-geogrid }}$ is the shear strength of the soil-geogrid interface, and $t_{\text {soil }}$ is the shear strength of the unreinforced soil.

The same method was applied to determine the $\alpha$ coefficient of the peak and critical angle of the shear strength. The values of the interface's shear strength coefficient $\alpha$ for all the tested soils and geogrids are summarized in Fig. 7.

The results showed that the smallest values of the coefficient a were determined for Sample 1 and that the highest values of the coefficient a were determined for Sample 3. The values of the coefficient a for Samples 2 and 3 are similar to each other for both geogrids tested. In the case of Sample 1, higher values of the coefficient a were obtained using the TG3030S geogrid. The results also showed that the values of the coefficient a for Samples 2 and 3 are higher in the critical stress state. The higher values of the coefficient a for the critical state have been also presented by, e.g., [17].

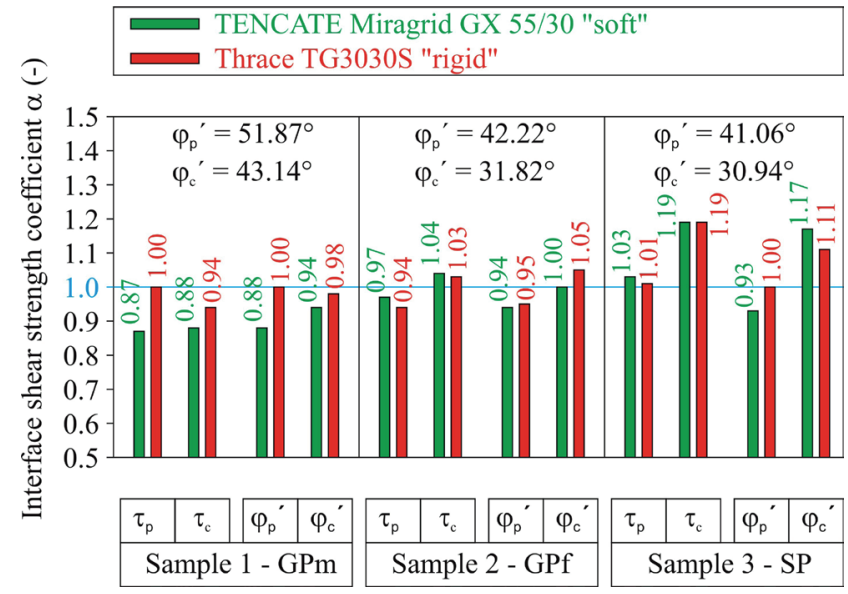

Fig. 7 Interface shear strength coefficients of tested soils with different geogrids

\section{Analysis of the results obtained}

The coefficient a increases with a decrease in the grain size. It can be assumed that the resistance of the transverse ribs causes this effect. The pore sizes are the largest for Sample 1 and the smallest for Sample 3. For this reason, the mobilization of the resistance of the transverse ribs is more effective for Sample 3 than for Sample 1. The assumptions are shown in Fig. 8. The change in the interface coefficient a with horizontal movement, mostly affected by the resistance of the transverse ribs, is shown in Fig. 9. The value of the coefficient a has a tendency to increase with horizontal movement. This effect is most visible in the case of Sample 3. The most important parameters that affect the soil-geogrid interface's shear strength are the grain sizes of the soil tested and the geometry of the geogrid.

The results of the measurements showed that the coefficient a is smaller than or equal to the value of 1.0 in the peak stress state (Fig. 7). Only in the case of Sample 3

Table 3 Shear strength properties determined from the testing

\begin{tabular}{|c|c|c|c|c|c|c|c|}
\hline Reinforcement & Property / Paran & & & Unit & Sample 1 & Sample 2 & Sample 3 \\
\hline \multirow{4}{*}{ No geogrid } & \multirow{2}{*}{ Angle of shear strength } & Peak & $\varphi_{p}^{\prime}$ & $\circ$ & 51.87 & 42.22 & 41.06 \\
\hline & & Critical & $\varphi_{c}^{\prime}$ & $\circ$ & 43.14 & 31.82 & 30.94 \\
\hline & \multirow{2}{*}{ Initial shear strength } & Peak & $\tau_{0, p}{ }^{\prime}$ & $\mathrm{kPa}$ & 0 & 1.03 & 2.94 \\
\hline & & Critical & $\tau_{0, c}^{\prime}$ & $\mathrm{kPa}$ & 1.14 & 2.9 & 1.16 \\
\hline \multirow{4}{*}{ GX55/30 } & \multirow{2}{*}{ Angle of shear strength } & Peak & $\varphi_{p}{ }^{\prime}$ & $\circ$ & 45.69 & 39.78 & 38.25 \\
\hline & & Critical & $\varphi_{c}^{\prime}$ & $\circ$ & 40.43 & 31.8 & 36.23 \\
\hline & \multirow{2}{*}{ Initial shear strength } & Peak & $\tau_{0, p}{ }^{\prime}$ & $\mathrm{kPa}$ & 5.97 & 4.43 & 10.83 \\
\hline & & Critical & $\tau_{0, c}^{\prime}$ & $\mathrm{kPa}$ & 0 & 4.64 & 0.11 \\
\hline \multirow{4}{*}{ TG3030S } & \multirow{2}{*}{ Angle of shear strength } & Peak & $\varphi_{p}{ }^{\prime}$ & $\circ$ & 51.96 & 39.98 & 40.98 \\
\hline & & Critical & $\varphi_{c}^{\prime}$ & $\circ$ & 42.43 & 33.3 & 34.2 \\
\hline & \multirow{2}{*}{ Initial shear strength } & Peak & $\tau_{0, p}{ }^{\prime}$ & $\mathrm{kPa}$ & 0.99 & 1.61 & 3.81 \\
\hline & & Critical & $\tau_{0, c}^{\prime}$ & $\mathrm{kPa}$ & 0 & 1.91 & 3.85 \\
\hline
\end{tabular}



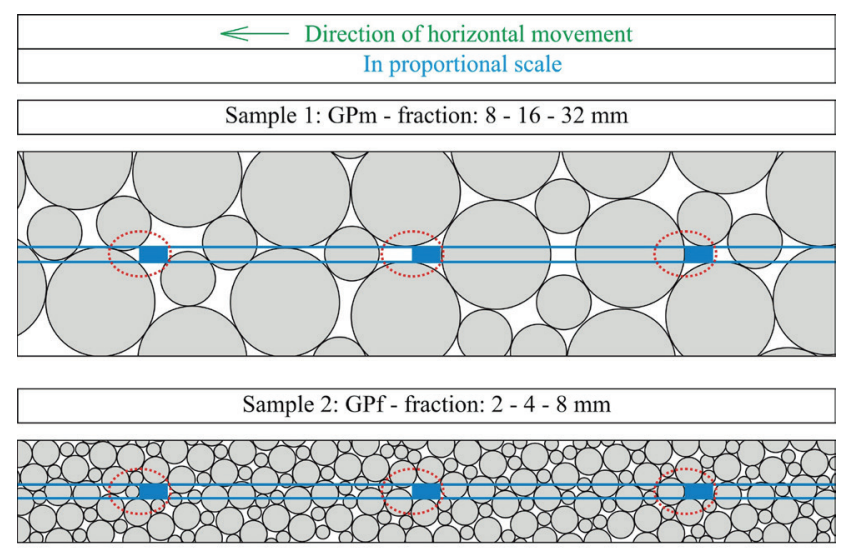

Sample 3: SP - fraction: 0.5 - 1 - $2 \mathrm{~mm}$

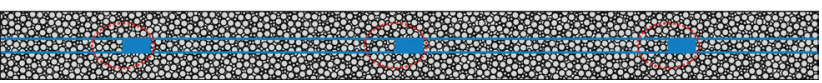

Fig. 8 Cross-section scheme of the TG3030S geogrid filled with grains of the soil tested
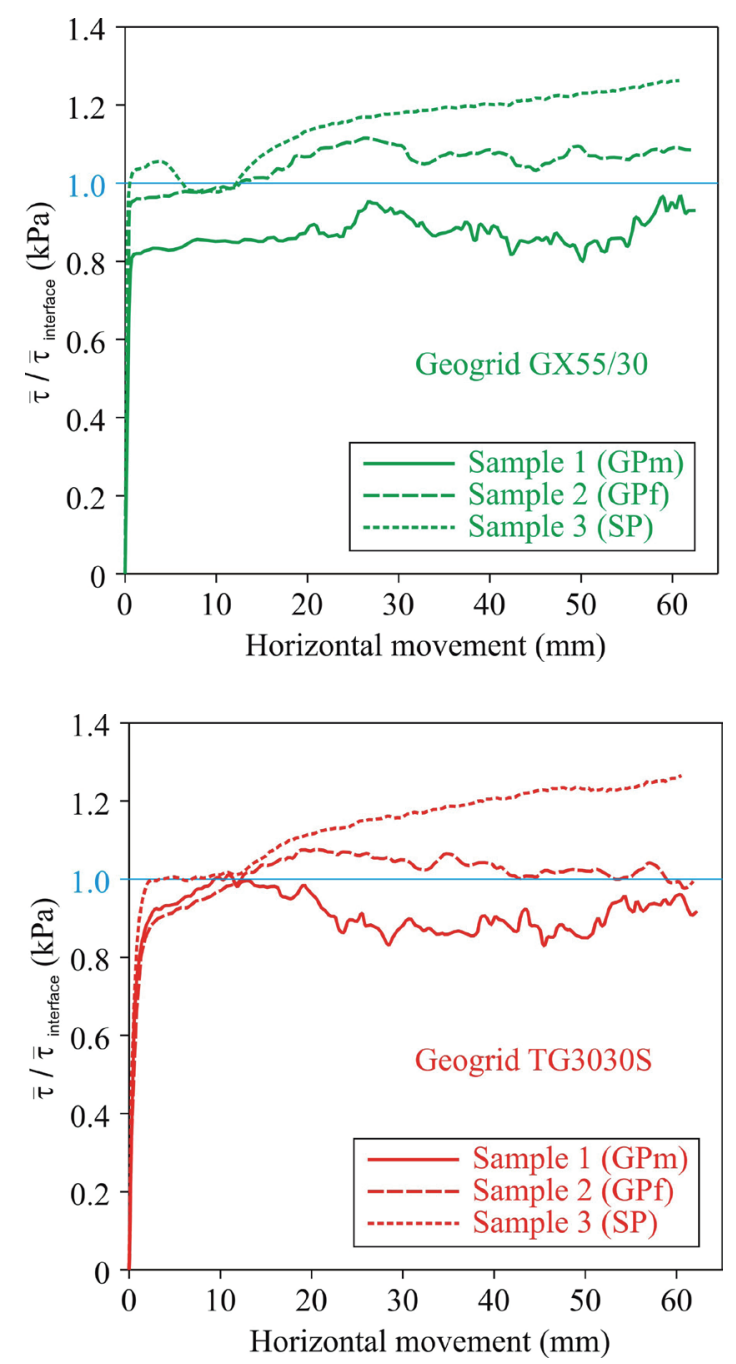

Fig. 9 Changes in of the interface coefficient a with horizontal movement was the value of coefficient a a little bit higher than 1.0 $(\alpha=1.03$ for the GX55/30 geogrid and $\alpha=1.01$ for the TG3030S geogrid). It can be assumed that the resistance of the transverse ribs was not mobilized enough because the horizontal movement was small. For this reason, the interface's shear strength for the peak stress state cannot be significantly improved. A deeper analysis is difficult because the peak values of the shear strength correspond to the different values of $I_{D}$ and the void ratio $e$. More important is the change in the interface's shear strength in the critical stress state. The samples have the same value of $I_{D}$, which corresponds to the critical void ratio ecrit. The resistance of the transverse ribs is mobilized due to the greater horizontal movement.

The change in the interface's shear strength coefficient $\alpha$ with a ratio of $d_{50} / h_{\text {geogrid }}$ is shown in Fig. 10, where the hgeogrid is the thickness of the transverse rib (Table 2). The results showed that the value of the coefficient a decreases with an increase in the ratio of $d_{50} / h_{\text {geogrid }}$. The optimal value of the ratio of $d_{50} / h_{\text {geogrid }}$ is reached at the point when the coefficient a equals 1.0. The values determined are as follows: $d_{50} / h_{\text {geogrid }}=3.2$ for the TG3030S geogrid and $d_{50} / h_{\text {geogrid }}=5.1$ for the GX55/30 geogrid. The most optimal grain size distributions for the geogrids tested are soils that have the parameter $d_{50}=6.4 \mathrm{~mm}$ for the TG3030S geogrid and $d_{50}=5.1 \mathrm{~mm}$ for the GX55/30 geogrid. The results presented in Fig. 10 also allow for the selection of the optimal thickness of a geogrid for the given soil.

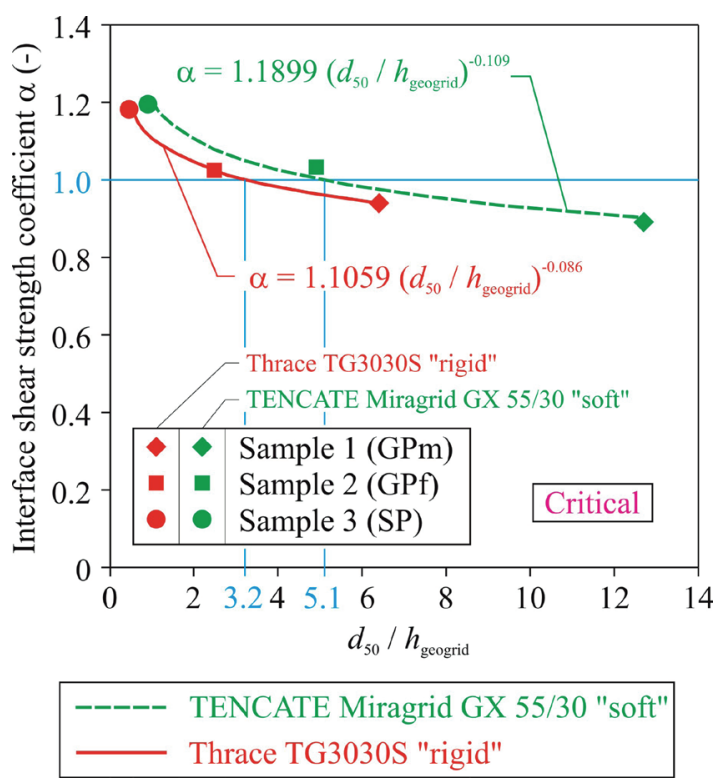

Fig. 10 Interface's shear strength coefficient, depending on the ratio of

$$
d_{50} / h_{\text {geogrid }}
$$




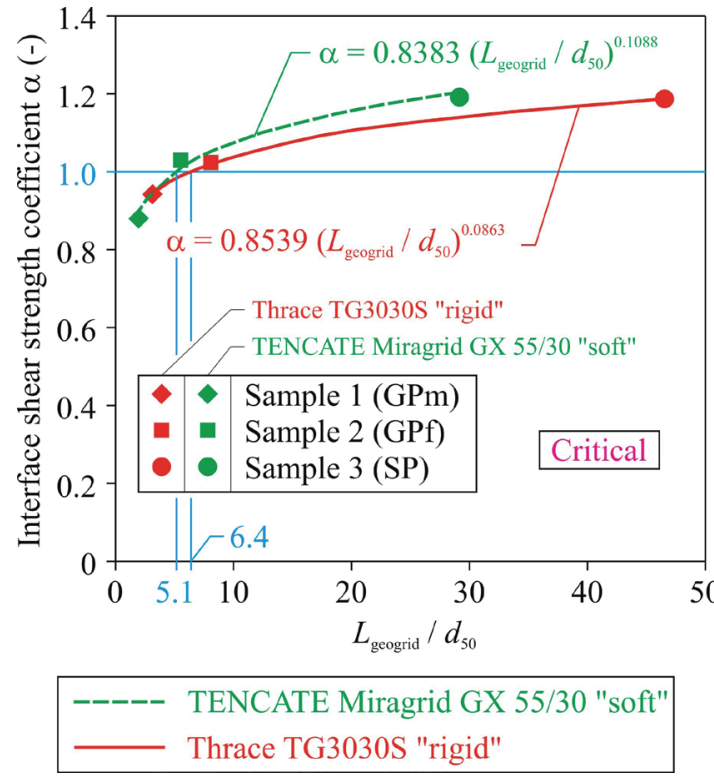

Fig. 11 Interface shear strength coefficient $\alpha$, depending on the ratio of

$$
L_{\text {geogrid }} / d_{50}
$$

The dependence of the interface's shear strength coefficient $\alpha$ on the ratio of $L_{\text {geogrid }} / d_{50}$ is shown in Fig. 11. The results are presented for the critical stress state when the resistance of the traverse ribs is fully mobilized. The $L_{\text {geogrid }}$ parameter represents the mesh size of the geogrid tested, see Table 2. The optimal value of the ratio of $L_{\text {geogrid }} / d_{50}$ is about 5.1 for the GX55/30 geogrid and about 6.4 for the TG3030S geogrid. Based on these results, the optimal value of $d_{50}$ for the GX55/30 geogrid is about $4.9 \mathrm{~mm}$ and about $6.25 \mathrm{~mm}$ for the TG3030S geogrid. The optimal values of the $d_{50}$ parameter, which were determined using two different methods, are compared in Table 4. The analysis shows that both diagrams, Fig. 10 and Fig. 11, can be used to determine the optimal value of the $d_{50}$ parameter. The shape of the grain curve must be suitable for the selected geogrid (the minimal and maximal grain sizes cannot exceed the limit values of the given type of geogrid).

\section{Conclusions}

The shear strength properties of the soil-geogrid interface using analytical as well as numerical methods must be taken into account in the design of reinforced earth structures. A reduction or increase in the soil-geogrid interface's shear strength is mostly defined by the interface's shear strength coefficient $\alpha$. This coefficient represents the ratio of the soil-geogrid interface's shear strength to the shear strength of the original unreinforced soil. The interface's shear strength was tested using the large-size
Table 4 Optimal value of the $d_{50}$ parameter for the geogrids tested

\begin{tabular}{lcc}
\hline & \multicolumn{2}{c}{$d_{50}(\mathrm{~mm})$} \\
Dependence & GX55/30 & TG3030S \\
\hline$d_{50} / h_{\text {geogrid }}$ for $\alpha=1.0$ & 5.1 & 6.40 \\
$L_{\text {geogrid }} / d_{50}$ for $\alpha=1.0$ & 4.9 & 6.25 \\
Average & 5.0 & 6.33 \\
\hline
\end{tabular}

SHEARMATIC 27-WF 2304 shear test apparatus. The tests were executed on three soil samples: poorly graded medium gravel (Sample 1), poorly graded fine gravel (Sample 2) and poorly graded coarse sand (Sample 3). The soils were reinforced using two types of geogrid, i.e., the TENCATE Miragrid GX55/30, a woven geogrid made of polyester (PET), and the Thrace TG3030S, an extruded stiff polypropylene geogrid (PP).

The interface coefficient $\alpha$ was smaller or at most close to the value of 1.0 in the peak stress state. The resistance of traverse ribs, which improves this value, was not mobilized enough because the horizontal movement is small. For this reason, it is not possible to achieve any significant improvement in the interface's shear strength at the peak stress sate. A deeper analysis is difficult because the peak values of the shear strength correspond to the different values of $I_{D}$ and the void ratio $e$. The value of the coefficient $\alpha$ can significantly increase in the critical stress state, depending on the grain size and geometry of the geogrid. At the critical stress state, the samples have the same value of $I_{D}$, which corresponds to the critical void ratio $e_{\text {crit }}$. It allowed for a deeper analysis. The interface's shear strength coefficient $\alpha$ reached its highest values in the case of Sample 3 and the smallest in the case of Sample 1, which means that the coefficient $\alpha$ increases by a decrease in the grain size as well as the pore sizes. The increase in coefficient $\alpha$ was mostly caused by the mobilization of the resistance of the transverse ribs of the geogrid used.

The results of the testing allowed for determining the optimal grain size for the geogrids tested. The value of the $d_{50}$ parameter was determined using the dependencies of $\alpha-d_{50} / h_{\text {geogrid }}$ and $\alpha-L_{\text {geogrid }} / d_{50}$. The optimal averaged value of the $d_{50}$ parameter was about $5.0 \mathrm{~mm}$ for the GX55/30 geogrid and about $6.33 \mathrm{~mm}$ for the TG3030S geogrid. The results of the testing presented in the paper can be directly used for soils and geogrids of similar properties and parameters. The optimal grain size for a geogrid with different parameters and soils with different properties can be successfully determined using the method presented in the paper. 


\section{Acknowledgement}

This article was created with the support of the Ministry of Education, Science, Research and Sport of the Slovak

\section{References}

[1] Arulrajah, A., Abdullah, A., Bo, M. W., Bouazza, A. "Ground improvement techniques for railway embankments", Proceedings of the Institution of Civil Engineers - Ground Improvement, 162(1), pp. 3-14, 2009

https://doi.org/10.1680/grim.2009.162.1.3

[2] Vega-Meyer, R., Shao, Y. "Geogrid-Reinforced and Pile-Supported Roadway Embankment", presented at Geo-Frontiers 2005 Congress, Austin, TX, USA, Jan. 24-26, 2005.

https://doi.org/10.1061/40777(156)9

[3] $\mathrm{Hu}$, Y., Li, H., Wang, X., Wang, Q. A. "Application of Geogrid in Widening Highway Embankment", In: 11 th International Conference of Chinese Transportation Professionals (ICCTP), Nanjing, China, 2011, pp. 3059-3066.

https://doi.org/10.1061/41186(421)304

[4] Hangen, H., Beilke, O., Rahier, A., Wüstefeld, D. "Geogrid Reinforced Steep Slopes Subjected to Railway Loading - Case Study", presented at GEORAIL 2011 International Symposium, Paris, France, May, 19-20, 2011. [online] Available at: https://www. huesker.co.uk/knowledge/publications/scientific-reviewed-papers. html?detailID=50\&cHash=66 cf177490351a159672b0ec28f078d7

[5] Blackwood, T. W., Vulova, C. W. "Geogrid Reinforced Embankment Constructed over Peat Soils in Clark Country, Washington: Design and Field Performance", In: Airfield and Highway Pavements Specialty Conference 2006, Atlanta, GA, USA, 2006, pp. 317-328. https://doi.org/10.1061/40838(191)27

[6] Bonaparte, R., Christopher, B. R. "Design and Construction of Reinforced Embankmnets over Weak Foundations", Transportation Research Records, 1153, pp. 26-39, 1987. [online] Available at: http://onlinepubs.trb.org/Onlinepubs/trr/1987/1153/1153-004.pdf

[7] Palmeira, E. M., Antunes, L. G. S. "Large scale tests on geosynthetic reinforced unpaved roads subjected to surface maintenance", Geotextiles and Geomembranes, 28(6), pp. 547-558, 2010. https://doi.org/10.1016/j.geotexmem.2010.03.002

[8] Allen, T. M., Barthus, R. J. "Design and Performance of 6.3-m-High, Block-Faced Geogrid Wall Designed Using K-Stiffness Method", Journal of Geotechnical and Geoenvironmental Engineering, 140(2), 2014. https://doi.org/10.1061/(ASCE)GT.1943-5606.0001013

[9] Dolinajová, K., Sňahničan, J. "Oporné múry vystužené geomrežami a dvojzákrutovou ocel'ovou siet'ou vystavené vysokému zat’aženiu v seizmickej oblasti", (Retaining walls reinforced with geogrids and double-twisted steel mesh exposed to high loads in the seismic area), In: Proceedings of the 12th Slovak Geotechnical Conference, 55 Years of Geotechnical Engineering in Slovakia, Bratislava, Slovakia, 2015, pp. 79-87.

[10] Omar, M. T., Das, B. M., Puri, V. K., Yen, S. C. "Ultimate bearing capacity of shallow foundations on sand with geogrid reinforcement", Canadian Geotechnical Journal, 30(3), pp. 545-549, 1993. https://doi.org/10.1139/t93-046
Republic within the VEGA grant No. 1/0530/19 and the VEGA grant No. 1/0842/18.

[11] Palmeira, E. M., Milligan, G. W. E. "Scale effects in direct shear tests on sand", In: Proceedings of the XII International Conference on Soil Mechanics and Foundation Engineering, Rio de Janeiro, Brazil, 1989, pp. 739-742. [online] Available at: https://www. researchgate.net/publication/285535248_Scale_effects_in_direct_ shear_tests_on_sand

[12] Farrag, K., Acar, Y. B., Juran, I. "Pull-out resistance of geogrid reinforcements", Geotextiles and Geomembranes, 12(2), pp. 133-159, 1993.

https://doi.org/10.1016/0266-1144(93)90003-7

[13] Liu, C.-N., Ho, Y.-H., Huang, J.-W. "Large scale direct shear tests of soil/PET-yarn geogrid interfaces", Geotextiles and Geomembranes, 27(1), pp. 19-30, 2009.

https://doi.org/10.1016/j.geotexmem.2008.03.002

[14] Zekkos, D., Athanasopoulos, G. A., Bray, J. D., Grizi, A., Theodoratos, A. "Large-scale direct shear testing of municipal solid waste", Waste Management, 30(8-9), pp. 1544-1555, 2010. https://doi.org/10.1016/j.wasman.2010.01.024

[15] Bakeer, R. M., Said, S. M., Cates, P., Subramanian, R. "Pullout and shear tests on geogrid reinforced lightweight aggregate, Geotextiles and Geomembranes", 16(2), pp. 119-133, 1998. https://doi.org/10.1016/S0266-1144(97)10025-5

[16] Lee, K. M., Manjunath, V. R. "Soil-geotextile interface friction by direct shear tests", Canadian Geotechnical Journal, 37(1), pp. 238252, 2000.

https://doi.org/10.1139/t99-124

[17] Umashankar, B., Chennarapu, H., Sasanka Mouli, S. "Interface Properties of Metal-Grid and Geogrid Reinforcements with Sand", In: International Foundations Congress and Equipment Expo (IFCEE 2015), San Antonio, TX, USA, 2015, pp. 1430-1438. https://doi.org/10.1061/9780784479087.129

[18] Sayeed, M. M. A., Janaki Ramaiah, B., Rawal, A. "Interface shear characteristics of jute/polypropylene hybrid nonwoven geotextiles and sand using large size direct shear test", Geotextiles and Geomembranes, 42(1), pp. 63-68, 2014. https://doi.org/10.1016/j.geotexmem.2013.12.001

[19] Koerner, R. M., Wayne, M. H., Carroll, R. G., Jr. "Analytic behavior of geogrid anchorage", In: Proceedings of Geosynthetics'89 Conference, IFAI, San Diego, CA, USA, 1989, pp. 525-536.

[20] Cancelli, A., Rimoldi, P., Togni, S. "Frictional characteristics of geogrids by means of direct shear and pullout tests", In: Proceedings of the International Symposium on Earth Reinforcement Practice, Kyushu, Fukuoka, Japan, 1992, pp. 29-34.

[21] Abu-Farsakh, M., Coronel, J., Tao, M. "Effect of Soil Moisture Content and Dry Density on Cohesive Soil-Geosynthetic Interactions Using Large Direct Shear Tests", Journal of Materials in Civil Engineering, 19(7), pp. 540-549, 2007. https://doi.org/10.1061/(ASCE)0899-1561(2007)19:7(540) 
[22] Xu, Y., Williams, D. J., Serati, M., Vangsness, T. "Effects of Scalping on Direct Shear Strength of Crusher Run and Crusher Run/Geogrid Interface", Journal of Materials in Civil Engineering, 30(9), Article number: 04018206, 2018. https://doi.org/10.1061/(ASCE)MT.1943-5533.0002411

[23] Sweta, K., Hussaini, S. K. K. "Effect of shearing rate on the behavior of geogrid-reinforced railroad balast under direct shear conditions", Geotextiles and Geomembranes, 46(3), pp. 251-256, 2018. https://doi.org/10.1016/j.geotexmem.2017.12.001

[24] Tuna, S. C., Altun, S. "Mechanical behaviour of sand-geotextile interface", Scientia Iranica, 19(4), pp. 1044-1051, 2012. https://doi.org/10.1016/j.scient.2012.06.009

[25] Arulrajah, A., Rahman, M. A., Piratheepan, J., Bo, M. W., Imteaz, M. A. "Evaluation of Interface Shear Strength Properties of Geogrid-Reinforced Construction and Demolition Materials Using a Modified Large-Scale Direct Shear Testing Apparatus", Journal of Materials in Civil Engineering, 26(5), pp. 974-982, 2014. https://doi.org/10.1061/(ASCE)MT.1943-5533.0000897

[26] Berg, R. R., Christopher, B. R., Samtani, N. C. "Design and Construction of Mechanically Stabilized Earth Walls and Reinforced Soil Slopes - Volume I", U. S. Department of Transportation Federal Highway Administration, Washington, DC, USA, Rep. FHWANHI-10-024, 2009. [online] Available at: https://www.fhwa.dot. gov/engineering/geotech/pubs/nhi10024/nhi10024.pdf

[27] Geng, M., Li, P., Li, J. "Numerical Analysis of a Geogrid-Reinforced High Embankment", In: Fifth International Conference on Transportation Engineering, Dalian, China, 2015, pp. 1074-1083. https://doi.org/10.1061/9780784479384.135
[28] Peng, F., Li, F., Tan, Y. "FEM Simulation of Deformation and Strength Characteristics in Geogrid-Reinforced Sand Retaining Wall under the Change of Loading Rate", In: Geo-Frontiers Congress 2011, Dallas, TX, USA, 2011, pp. 3807-3817. https://doi.org/10.1061/41165(397)389

[29] Sulovska, M., Stacho, J. "Determination of shear strength properties of coarse $\square$ grained materials for stone column", In: XVI DECGE 2018 Proceedings of the 16th Danube $\square$ European Conference on Geotechnical Engineering, Skopje, Macedonia, 2018, pp. 791-796. https://doi.org/10.1002/cepa.767

[30] Stacho, J., Sulovska, M. "Determination of the density of stone columns using in-situ testing", In: 17th International Multidisciplinary Scientific GeoConference SGEM 2017, Albena, Bulgaria, 2017, pp. 223-230.

https://doi.org/10.5593/sgem2017/12/S02.029

[31] GeoMat "Miragrix GX", [online] Available at: https://www.geomat. sk/miragrid-gx/ [Accessed: 15 January 2020]

[32] Thrace Group "Geogrids \& Geocomposites/TG3030S", [online] Available at: https://m.thracegroup.com/ua/en/technical-fabrics/geosynthetics/geogrids/\#!/products:420 [Accessed: 15 January 2020]

[33] Wu, Y., Wen, L. "Simple Modelling of Undrained Shear Response of Granular Materials", Periodica Polytechnica Civil Engineering, 62(4), pp. 947-955, 2018. https://doi.org/10.3311/PPci.11996 